\title{
DIE ARZNEI-GEWICHTE.
}

(Nach Galen, Ausg. v. Kühn, XIX, S. 752.)

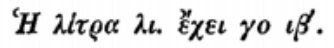

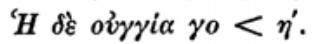

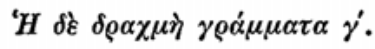

$T \delta \delta \varepsilon \bar{\varepsilon} \gamma \rho \dot{\alpha} \mu \mu \alpha \rightarrow \delta \beta o \lambda o \dot{v} \varsigma \beta^{\prime}$.

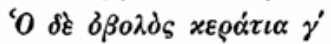

iे $\chi \alpha \lambda x o \tilde{v} \varsigma \eta^{\prime}$.
Das Pfund enthält 12 Unzen.

Die Unze 8 Drachmen.

Die Drachme 3 Scrupel.

Der Scrupel 2 Obolen.

Der Obolus 3 Schoten

oder 8 Gran. [Also 1 Scr. = 16 Gran.]

Das Gramma $\left(-\frac{1}{24}\right.$ Unze) ist nicht erheblich verschieden von unsrem Gramm. $\left(\zeta^{j} \mathrm{j}=30,0.\right)$

Bei uns holt der Kranke die Arznei aus der Apotheke. Bei den Alten verfertigte der Arzt die Arznei und wandte sie an. Deshalb steht am Schluss der griechischen Arzneivorschrift $\chi \rho \tilde{\omega}$ "gebrauche es"; statt unseres $d a$, ,gieb es".

\section{BERICHTIGUNGEN.}

Seite 2, Zeile 14, lies $x v-x \lambda o \tau \varepsilon \rho \varepsilon \tilde{\text {. }}$

S. 12, Z. 6, lies $\pi \varepsilon \varrho \iota \varepsilon \tau \alpha \mu \varepsilon ́ v \eta$.

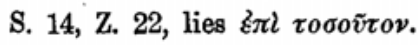

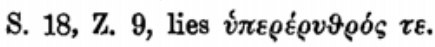

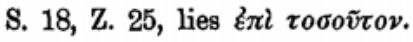

S. 24 , Z. 18 , lies ' $H \rho \alpha$.

S. 28, Z. 9 , streiche $\chi \varrho \eta ́$.

S. 28 , Z. 15 lies $\dot{\alpha} \mu \beta \lambda \tilde{v} v \alpha \iota$ und $\gamma \lambda v x \tilde{\alpha} v \alpha \iota$.

S. 30, Z. 15 , lies $\pi \varrho \dot{\partial} \beta \rho \alpha \chi \varepsilon \dot{\varepsilon} \omega \nu$.

S. 36, Z. 9, lies 'Eגxoṽ $\alpha$. 